\title{
Understanding the Presentations and Patterns of Traumatic Spinal Cord Injuries to Develop the Data Collection Format
}

\author{
Ranabir Pal ${ }^{1}$ Mithasha Singh ${ }^{2}$ Kiran Kumar ${ }^{3}$ Vinay Sagar Sharma ${ }^{4} \quad$ Mundlapudi Jahanavi $^{5}$ \\ Ranjan Kumar Jena ${ }^{5}$ Ashok Munivenkatappa ${ }^{6} \quad$ Prashant Bhandarkar $^{7} \quad$ Amit Agrawal $^{5}$
}

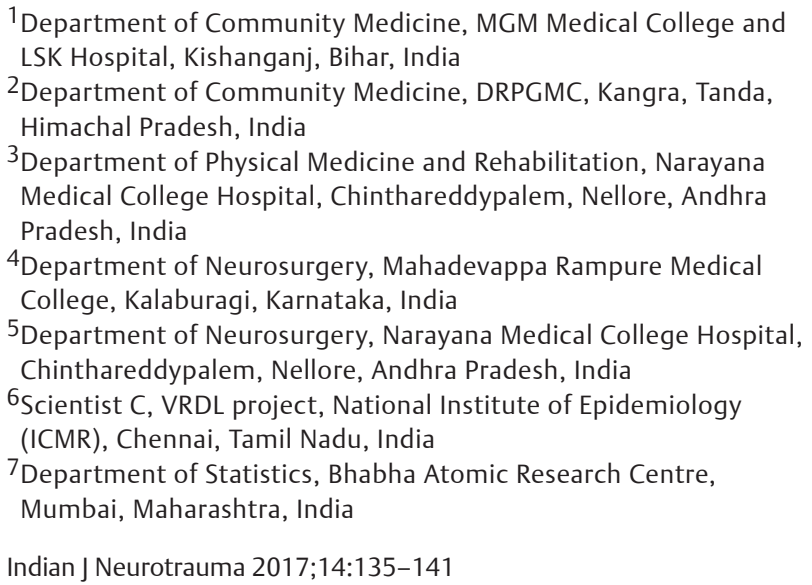

Address for correspondence Amit Agrawal, MCh, Department of Neurosurgery, Narayana Medical College Hospital, Chinthareddypalem, Nellore 524003, Andhra Pradesh, India (e-mail: dramitagrawal@gmail.com).

\begin{abstract}
Keywords

- spinal injury

- data collection

- traumatic spinal cord injury

Background The intensity of the damage to the nerve fibers is not measured through the severity of the spinal cord injury.

Objective To understand the pattern of neurologic features in traumatic spinal cord injuries.

Methods This was a case series of the total care of patients with consecutive acute spinal cord injury at different levels during the years 2015 to 2017, from extrication and transportation following the accident to death, or the completion of primary definitive rehabilitation. This work examined demographic and clinical characteristics of 220 consecutive cases of acute spinal injuries with or without neurologic symptoms subjected to conservative and surgical interventions. Age, sex, addiction patterns of smoking and alcohol, presenting symptoms, neurologic status, and postoperative outcomes are compared with the preoperative findings.

Results The registry included 220 patients with spinal injury; majority in 41 to 50 years age group (28.6\%) and males (77.7\%). Nearly one-half had cervical (46.3\%) injuries; $84.3 \%$ male; comparable proportion of dorsal and lumbar injuries had male dominance. One-fifth of the patients were smokers and alcoholics, all males. Mean hospital stay was $36.7 \pm 140.5$ days. Majority presented with neck pain (48.9\%), radicular pain (56.4\%), limb weakness (73.3\%), and tingling sensation (47.7\%); $14.4 \%$ reported tightness in limbs and $18.8 \%$ bladder involvement. In postoperative period, symptoms persisted in varied proportions. Significantly of postoperative neck pain was observed more among males (88.5\%), yet symptoms that reduced postoperatively were neck pain, limb weakness, and tingling sensation. However, bladder involvement increased significantly.

Conclusion The results showed that greater attention should be paid to older and male population that has more spinal cord injuries while comparable to those of the other studies in neurodeficits and clinical features.
\end{abstract}




\section{Introduction}

Spinal injuries secondary to trauma are a major cause of morbidity, mortality, and disability leading to significant health care expenditure. Increases in traffic safety standards and improved health care resources may have changed the characteristics and incidence of spinal injury. The chief line of the nerve tissues connecting the brain and vertebral canal is the spinal cord where the spinal nerves surface. When a spinal cord gets injured, it sometimes leads to permanent damage to bodily functions. Injury to the spinal cord occurs when a major damage to any spinal nerve occurs at the spinal canal. When the damage that occurs to the spinal cord is unsurpassable, defects in the sensory, motor, and reflex messages are detected after a spinal cord injury (SCI). Going by the context, a person will not be able to function properly and the condition worsens when the injury level is higher with varied clinical presentation with trouble with walking and proper movement; inability to differentiate sensations and feel heat, cold, and touch; loss of the control of the bowel or bladder movements; moments of inflated reflex actions or seizures; unnatural sexual function and sensitivity; moments of growing numbness and tingling to extreme; signs of breathing problems; and coughing among others. The most common cause of spinal injury is trauma, yet nontraumatic SCI may occur because of arthritis, cancer, inflammation, infections, or spinal disk deterioration. ${ }^{1}$ Spinal cord lesions have various etiologies, and trauma is one of the leading causes. Patients with SCIs often have motor, sensory, and autonomic dysfunctions, and require a multidisciplinary rehabilitation program. ${ }^{2}$ In this scenario, this study was conducted to understand the pattern of neurologic features in traumatic SCIs to assess the changing characteristics and spectrum of clinical presentations of SCIs over a period.

\section{Material and Methods}

This was a case series of the total care of consecutive acute SCI at different levels during the years 2015 to 2017, from extrication and transportation following the accident to death, or the completion of primary definitive rehabilitation. This work examined demographic and clinical characteristics of 220 consecutive cases of acute spinal injuries with or without neurologic symptoms subjected to conservative and surgical interventions. Age, sex, addiction patterns of smoking and alcohol, presenting symptoms, neurologic status, and postoperative outcomes are compared with the preoperative findings. Standard operative procedures were followed with optimized approach of combined conservative and surgical interventions for best possible outcomes following Institutional ethical standards. Age, sex, addiction patterns of smoking and alcohol, presenting symptoms, neurologic status, and postoperative outcomes are compared with the preoperative findings. After approval from the institutional ethical committee, the medical records of patients admitted and managed for traumatic SCI were collected in a predesigned proforma. The study included data over a period of 3 years -2 year retrospective and 1 year prospective. The detailed information about patients included sex, age, and injury level; neurologic deficits were grouped by level: cervical, thoracic, lumbar, and sacral segment.

\section{Statistical Analysis}

The data were analyzed using SPSS version 24 (IBM Inc.) for windows. The descriptive statistics was calculated by mean and frequencies of data. Incidence and prevalence, as well as male/female ratio, causes of accidents, and their relationship to sex, race, age, vertebral level, associated injuries, and profession, were discussed. The motor scores on each upper and lower limb joints were added, and the output was presented as median (Interquartile range) upper/lower extremity scores. After checking for normality of distribution of the scores, relevant nonparametric test (Wilcoxon signed rank test) to test the difference between pre- and postoperative scores was applied. To implement preventive programs purposefully, the relationships among age, sex, occupation, level, severity, and etiology were analyzed descriptively.

\section{Results}

The registry included 220 patients with spinal injury. Majority were $(77.7 \%$ ) males, and $28.6 \%$ in 41 to 50 years age group. Majority of young patients were males. Around one-half of the injuries were cervical (46.3\%), of which $84.3 \%$ were in male patients. Almost equal proportion of dorsal and lumbar injuries was observed with the same pattern of male dominance. This distribution was statistically significant $(p=0.02)$. Smoking was prevalent among $19.5 \%$ patients and alcohol among $18.6 \%$. All the smokers and alcohol consumers were males, and this distribution was statistically significant $(p=0.00)$. Mean hospital stay was $36.7 \pm 140.5$ days, and there was no significant difference between male and female patients ( - Table $\mathbf{1}$ ). Around one-half (48.9\%) of the preoperative symptoms comprised neck pain, $56.4 \%$ with radicular pain, $73.3 \%$ with limb weakness, $47.7 \%$ with tingling sensation, $14.4 \%$ with tightness in limbs, and $18.8 \%$ with bladder involvement. All the preoperative symptoms persisted in postoperative period in varied proportions. Neck pain decreased from $48.9 \%$ preoperatively to $44.8 \%$ postoperatively $(p=0.04)$. Limb weakness preoperatively decreased significantly from 73.3 to $60.1 \%$ postoperatively $(p=0.02)$. Another symptom that reduced in significant proportion was tingling sensation $(47.7-25.2 \% ; p<0.001)$. However, bladder involvement increased significantly from 18.8 to $33.6 \%$ postoperatively $(p=0.01)$ ( - Table 2 ).

Out of the 220 patients with spinal injury, 195 underwent surgery. The motor, sensory, and deep tendon reflexes (DTRs) eliciting the spinal level involvement pre- and postoperatively are depicted in - Table 3. The highest motor score is 40 that denotes the active movement of muscle against full gravity, and lowest score is 0 that denotes total paralysis. The median motor score for upper right extremity reduced significantly from 36 (interquartile range [IQR], 8) preoperative to 32 (IQR, 9.5) postoperative $(p=0.02)$. There was a similar nonsignificant reduction in median scores in the left upper extremities of registered patients. There was no change postoperatively in median scores of both right and left lower extremities of registered patients. 
Table 1 Spinal cord level involvement among the study population subjected to injury

\begin{tabular}{|c|c|c|c|c|c|}
\hline & & \multicolumn{2}{|c|}{ Gender } & \multirow[t]{2}{*}{ Total } & \multirow[t]{2}{*}{$p$ Value } \\
\hline & & Female & Male & & \\
\hline \multirow[t]{2}{*}{ Total } & & 49 & 171 & 220 & \\
\hline & & $22.3 \%$ & $77.7 \%$ & $100.0 \%$ & \\
\hline \multirow[t]{8}{*}{ Age range } & $0-10$ & 0 & 2 & 2 & 0.87 \\
\hline & $11-20$ & 2 & 9 & 11 & \\
\hline & $21-30$ & 6 & 28 & 34 & \\
\hline & $31-40$ & 11 & 31 & 42 & \\
\hline & $41-50$ & 16 & 47 & 63 & \\
\hline & $51-60$ & 8 & 35 & 43 & \\
\hline & $61-70$ & 4 & 16 & 20 & \\
\hline & $>70$ & 2 & 3 & 5 & \\
\hline \multirow[t]{3}{*}{ Spinal level } & Cervical & 16 & 86 & 102 & 0.02 \\
\hline & Dorsal & 9 & 35 & 44 & \\
\hline & Lumbar & 17 & 30 & 47 & \\
\hline \multirow[t]{3}{*}{ Smoking } & No & 19 & 29 & 48 & 0.00 \\
\hline & Yes & 0 & 43 & 43 & \\
\hline & Not known & 16 & 39 & 55 & \\
\hline \multirow[t]{3}{*}{ Alcohol intake } & No & 15 & 17 & 32 & 0.00 \\
\hline & Yes & 0 & 41 & 41 & \\
\hline & Not known & 16 & 39 & 55 & \\
\hline Mean hospital stay in days $( \pm S D)$ & & $37.2 \pm 105.8$ & $36.6 \pm 149.1$ & $36.7 \pm 140.5$ & 0.98 \\
\hline
\end{tabular}

Abbreviation: SD, standard deviation.

Note: Values in bold are statistically significant.

The DTR scores in upper and lower limb were added up, resulting in maximum score of 9 that denotes exaggerated response in all the tendons and minimum of 0 that denotes absent reflex. The median score of DTR remained 6 in both upper and lower limbs. The significant change in pre- to postoperative in median (IQR) was observed in right lower extremity from $6(0)$ to $6(3)(p=0.01)$.

The proportion of altered sensory reflexes in right upper extremity was $10.6 \%$, which significantly reduced to nil postoperatively $(p<0.001)$; hence, there was significant increase in normal reflexes. There was also an increase in proportion of normal sensory reflex in left upper extremity from pre- to postoperative. The proportion of altered sensory reflexes increased in lower extremity from pre- to postoperative registered patients; however, this was not statistically significant ( - Table 3 ).

\section{Discussion}

The registry included 220 patients with spinal injury, and the results were noted to be parallel to those of the other studies.

\section{Age}

In this study, majority of the patients (28.64\%) were in 41 to 50 years age group, followed by 51 to 60 and 31 to 40 years age group; both were little more than $19 \%$. In the Turkish study on $1,694 \mathrm{SCl}$ patients, more frequent were among those between the ages of 15 and 39 years. $^{2}$ A study from Iceland also reported that mean age of traumatic SCI cases was 39 years. $^{3}$ In a retrospectively reviewed hospital in China, the records of all patients aged 18 years or older with traumatic cervical spinal fracture of two university-affiliated hospitals between January 2001 and December 2010 noted that the mean age was $42.5 \pm 13.8$ years, with a range of 18 to 86 years. ${ }^{4}$ Literature supports that with increasing age, there was an increase in spinal injuries, frequency of blunt SCIs, and injuries at multiple spinal levels for which the underlying reasons may be anatomy, physiology, or mechanism. ${ }^{5}$ In the study on spinal trauma in Mainland China, approximately $79.32 \%$ of spinal trauma occurred in patients between 20 and 60 years of age. ${ }^{6}$ The study done in Tianjin, China, mean age of traumatic SCl as $46.0 \pm 14.2$ years (men $45.8 \pm 14.2$ years, women $47.5 \pm 14.5$ years), with a range of 16 to 90 years. $^{7}$

\section{Gender}

In most spinal injured cases in this series, there were (77.7\%) males and majority of younger age group were males. Of the cervical injuries, $84.3 \%$ were male cases; dorsal and lumbar 
Table 2 Distribution and comparison of pre- and postoperative symptoms

\begin{tabular}{|c|c|c|c|c|c|}
\hline Preoperative symptom & & Total & $\begin{array}{l}\text { Postoperative } \\
\text { symptom }\end{array}$ & Total & $\begin{array}{l}\text { Test of significance on } \\
\text { difference of proportions }\end{array}$ \\
\hline \multirow[t]{3}{*}{ Neck pain } & No & 47 & No & 28 & \\
\hline & Yes & 73 & Yes & 52 & 0.04 \\
\hline & Not known & 29 & Not known & 36 & \\
\hline Total & & 149 & & 116 & \\
\hline \multirow[t]{3}{*}{ Radicular pain } & No & 41 & No & 27 & \\
\hline & Yes & 87 & Yes & 58 & 0.26 \\
\hline & Not known & 26 & Not known & 32 & \\
\hline Total & & 154 & & 117 & \\
\hline \multirow[t]{3}{*}{ Limb weakness } & No & 36 & No & 17 & \\
\hline & Yes & 121 & Yes & 68 & 0.02 \\
\hline & Not known & 8 & Not known & 28 & \\
\hline Total & & 165 & & 113 & \\
\hline \multirow[t]{4}{*}{ Tingling sensation } & No & 44 & No & 50 & \\
\hline & Yes & 71 & Yes & 29 & $<0.001$ \\
\hline & Not known & 34 & Not known & 36 & \\
\hline & & 149 & & 115 & \\
\hline \multirow[t]{3}{*}{ Tightness in limbs } & No & 76 & No & 58 & \\
\hline & Yes & 20 & Yes & 13 & 0.50 \\
\hline & Not known & 43 & Not known & 43 & \\
\hline Total & & 139 & & 114 & \\
\hline \multirow[t]{3}{*}{ Bladder involvement } & No & 85 & No & 26 & \\
\hline & Yes & 29 & Yes & 38 & 0.01 \\
\hline & Not known & 40 & Not known & 49 & \\
\hline Total & & 154 & & 113 & \\
\hline \multirow[t]{3}{*}{ Other symptoms } & No & 57 & No & 50 & \\
\hline & Yes & 26 & Yes & 3 & $<0.001$ \\
\hline & Not known & 49 & Not known & 62 & \\
\hline Total & & 132 & & 115 & \\
\hline
\end{tabular}

injuries also showed same pattern of male dominance that was statistically significant. In the Turkish SCI study, more frequent trauma victims were males. ${ }^{2}$ The Iceland study reported males predominance $(73 \%)$ among traumatic SCI cases. ${ }^{3}$ In the Chinese study on traumatic cervical spinal fracture, the male/female ratio was 4.3:1. ${ }^{4}$ In the Canadian study on traumatic SCIs, men demonstrated predominantly lower cervical injuries and women more likely to exhibit upper cervical injuries. ${ }^{8}$ Mainland China research group on spinal injury noted that the injury had a male/female ratio of 2.33:1 and that mortality rate among male was also twice that of the female. ${ }^{6}$ The study from Tianjin, China, on epidemiology of traumatic SCI reported that male/female ratio was $5.63: 1 .^{7}$

\section{Spinal Level}

Around one-half of the injuries were cervical (46.3\%), followed by almost equal proportion of dorsal and lumbar injuries. Research group from Iceland on traumatic SCI observed that the injury was complete in $39 \%$; cervical lesions were $57 \%$ and thoracic/lumbar lesions were $43 \% .^{9}$ In the study reported from Mainland China, the lumbar spine was most frequently involved (56.09\%), followed by thoracic spine (23.77\%), cervical spine (17.75\%), and sacrococcygeal vertebrae (2.39\%). Fracture-dislocation occurred most frequently in the cervical and lumbar spines, whereas SCI occurred most frequently in the cervical and thoracic spines. Children younger than 10 years were prone to cervical spine injury, whereas adults older than 60 years were more prone to 
Table 3 Distribution of pre- and postoperative motor, sensory, and DTRs in upper and lower extremities

\begin{tabular}{|c|c|c|c|c|c|c|}
\hline & Right side of body & & & Left side of body & & \\
\hline & Preoperative & Postoperative & $p$ Value & Preoperative & Postoperative & $p$ Value \\
\hline $\begin{array}{l}\text { Median upper extremity } \\
\text { motor scores (IQR) }\end{array}$ & $36(8.00)$ & $32(9.50)$ & 0.02 & $35.5(8.00)$ & $32(8.00)$ & 0.08 \\
\hline $\begin{array}{l}\text { Median lower extremity } \\
\text { motor scores (IQR) }\end{array}$ & $32(16.00)$ & $32(22.00)$ & 0.07 & $32(22.00)$ & $32(17.75)$ & 0.54 \\
\hline $\begin{array}{l}\text { Median upper limb DTR } \\
\text { score (IQR) }\end{array}$ & $6(0)$ & $6(0)$ & 0.68 & $6(0)$ & $6(0)$ & 0.89 \\
\hline $\begin{array}{l}\text { Median lower limb DTR } \\
\text { score (IQR) }\end{array}$ & $6(0)$ & $6(3.00)$ & 0.01 & $6(1.00)$ & $6(2.00)$ & 0.18 \\
\hline Handgrip & Number (\%) & Number (\%) & $P$ value & Number (\%) & Number (\%) & $P$ value \\
\hline 0 & $6(4.7)$ & $5(4.8)$ & 0.95 & $6(4.3)$ & $4(3.5)$ & 0.28 \\
\hline Normal & 94 (72.9) & $84(80.8)$ & 0.16 & $105(75.5)$ & $92(80.7)$ & 0.33 \\
\hline $50-75 \%$ & $18(14)$ & $8(7.7)$ & 0.13 & $20(14.4)$ & $11(9.6)$ & 0.25 \\
\hline $25-50 \%$ & $8(6.2)$ & $7(6.7)$ & 0.87 & $4(2.9)$ & $7(6.1)$ & 0.20 \\
\hline $25 \%$ & $3(2.3)$ & 0 & 0.12 & $4(2.9)$ & 0 & 0.07 \\
\hline Total & $129(100)$ & $104(100)$ & & $139(100)$ & $114(100)$ & \\
\hline Upper limb sensory reflex & Number (\%) & Number (\%) & $p$ Value & Number (\%) & Number (\%) & $p$ Value \\
\hline Absent & $3(2.1)$ & $8(7.8)$ & $<0.001$ & $5(3.5)$ & 0 & 0.05 \\
\hline Normal & $123(87.2)$ & $94(92.2)$ & $<0.001$ & $120(85.2)$ & $94(92.2)$ & 0.09 \\
\hline Altered & $15(10.6)$ & 0 & $<0.001$ & $16(11.3)$ & $8(7.8)$ & 0.90 \\
\hline Total & $141(100)$ & $102(100)$ & & $141(100)$ & $102(100)$ & \\
\hline \multicolumn{7}{|l|}{ Lower limb sensory reflex } \\
\hline Absent & $8(5.7)$ & $4(4.2)$ & 0.62 & $8(5.8)$ & $4(3.9)$ & 0.49 \\
\hline Normal & $118(83.7)$ & $78(82.1)$ & 0.75 & $106(77.4)$ & $85(82.5)$ & 0.33 \\
\hline Altered & $15(10.6)$ & $13(13.7)$ & 0.48 & $23(16.8)$ & $14(13.6)$ & 0.50 \\
\hline Total & $141(100)$ & $95(100)$ & & $137(100)$ & $103(100)$ & \\
\hline
\end{tabular}

Abbreviations: DTR, deep tendon reflex; IQR, interquartile range.

osteoporotic thoracic and lumbar fractures. Lumbar spine injury was the most curable, whereas cervical spine injury was associated with the worst prognosis and the highest medical costs. ${ }^{6}$ The study done in Tianjin, China, on traumatic SCI reported that the lesion level was cervical in $71.5 \%$, thoracic $13.3 \%$ and lumbar/sacral $15.1 \%$; the frequency of tetraplegia $(71.5 \%)$ was higher than paraplegia $(28.5 \%)$, and roughly four-fifth of tetraplegia cases were incomplete injury. ${ }^{7}$ Researchers from the west reported that $65 \%$ of all cervical spine fractures and $80 \%$ of the multisegmental injuries were accompanied by an associated injury. The highest incidence of associated injuries was observed in patients with multilevel fractures (96.5\%). Patients with a concomitant injury were more likely to sustain a spinal cord lesion; $11.2 \%$ patients exhibited a complete motor and sensory deficit; $13.5 \%$ an incomplete and $75.3 \%$ no neurologic deficit. The highest number of complete motor and sensory neurologic deficits was found in cervical spine fractures (19.7\%). Most patients, 308 (54.8\%), sustained a compression fracture, 95 (16.9\%) patients a distraction fracture, and 104 (18.5\%) experienced a rotational fracture. ${ }^{10}$

\section{Smoking}

Smoking was prevalent among $19.5 \%$ patients, and all the smokers were males. This distribution was statistically significant.

\section{Alcohol Intake}

Consumption of alcohol was noted among $18.6 \%$ of SCI cases in this series, and all the alcohol consumers were males, which was statistically significant.

\section{Mean Hospital Stay}

In this study, mean hospital stay was $36.7 \pm 140.5$ days, and there was no significant sex difference in stay. In the Canadian study, increasing rates of surgical treatment were not associated with improvements in mortality rate or length of hospital stay. Patients older than 75 years who presented with acute traumatic SCIs had a mortality rate of one in five while in hospital. ${ }^{8}$

\section{Distribution and Comparison of Pre- and Postoperative Symptoms}

All the preoperative symptoms persisted in postoperative period in varied proportions. 


\section{Neck Pain}

Around one-half of the preoperative symptoms (48.9\%) comprised neck pain whereas majority of postoperative neck pain was observed among males (88.5\%), and this distribution was statistically significant.

\section{Radicular Pain}

Of the preoperative symptoms, 56.4\% complained with radicular pain that persisted in varied proportions postoperatively.

\section{Limb Weakness}

Of the preoperative presenting symptoms, 73.3\% had limb weakness that decreased significantly to $60.1 \%$ postoperatively.

\section{Tingling Sensation}

Preoperative in this series, $47.7 \%$ presented with tingling sensations that reduced in significant proportion to $25.2 \%$.

\section{Tightness in Limbs}

Preoperatively, $14.4 \%$ of patients complained of tightness in limbs that persisted postoperatively in varied proportions.

\section{Bladder Involvement}

Preoperatively, $18.8 \%$ patients showed signs and symptoms of urinary bladder involvement; however, bladder involvement increased significantly from 18.8 to $33.6 \%$ postoperatively.

\section{Distribution of Movements, Grips, and Reflexes: Pre- and Postoperative}

In patients of this registry, the right upper extremity motor scores were preoperatively in majority demonstrating active movement against some resistance. The median scores, however, reduced (became poorer) significantly postoperatively, corroborating with the dominance of cervical spine injuries. Contrast findings were observed in case of sensory reflexes that improved postoperatively in upper extremities and deteriorated in lower extremities. The authors are yet to find any published literature with details of clinical features of movements, grips, and reflexes: pre- and postoperative for traumatic SCIs to compare theirs.

In search of a model of care, future care of acute SCIs information was extracted from the available ambulance records, and the patients and many of the responsible physicians were interviewed personally. The study was detailed and intensive, and it included a review of each patient's hospital records in each hospital up to discharge from the rehabilitation program into the community or to a chronic care unit. The data were compiled in accordance with a detailed and lengthy questionnaire developed for this study. The incidence of acute cord injuries in Ontario in 1969 and 1970 amounted to $244-$ in $1969,15.9$ per million population and in 1970 , 13.6 per million. As in other studies road accidents took first place, followed by falls from a height; sports injuries ranked third and $65.7 \%$ of these were caused by diving into shallow water. Age incidence and incidence by month, day of week, and time of day were identified. Fridays and Saturday afternoons in July and August are particularly hazardous. The study continued to the end of 1974 by which time 34 deaths had been recorded. Peak incidence of death occurred within 14 days of injury. The most common cause of death was respiratory in origin. Geographical distribution and the type of hospital treating the acutely injured patient were identified. Fourteen percent of persons with spinal column injury suffered progressive or sequential spinal cord damage both prior to and following medical contact. The incidence of pressure sores and genitourinary sepsis and calculosis was high in all types of hospitals. The effect of operative treatment was noted in cases of complete quadriplegia and paraplegia. Of the 133 survivors who undertook a rehabilitation program, $84 \%$ returned to their homes and 59\% achieved gainful employment or ongoing education. The cost was determined of general hospital services and rehabilitation programs. A new model for the care of the SCI patients in Ontario was proposed. ${ }^{11}$

\section{Preventive Strategies}

This registry demonstrated that majority of the spinal injury was sustained by males, in age group 31 to 60 years. History of alcohol intake was present in around one-fifth of patients and that to among males only. Preventive strategies need to focus on this demographic profile that is more prone to sustain road traffic injuries or falls leading to spinal injuries. Most patients had cervical lesions. Emergency prehospital care is crucial, as the lesions are potentially life threatening and can have devastating functional consequences. The possibility of spinal cord trauma must always be kept in mind, and the spine must be immobilized. Training of paramedics and general public who play a significant role in transferring the patient from the point of accident to emergency will be a major preventive step. Immobilizing and proper handling of the patient save many other injuries of spine. The initial neurologic examination is extremely important.

\section{Strengths of This Study}

This study provided a unique opportunity to collect meaningful data on the variations of clinical features related to SCIs and help identify the variables for the creation of an SCI registry.

\section{Limitations of This Study}

The authors had several limitations. First, this was single-center study with limited external validity. Further, they could not reach robust sample size at this phase of study.

\section{Future Directions of This Study}

To the authors' knowledge, many studies report on the epidemiology of SCIs, yet national level representative study of SCI has not been performed in India while the number is empirically estimated to be large and would increase gradually similar to other developing countries. The authors have plan for prospective long-term studies in the dedicated setting with precise recording of their baseline neurologic impairment in the next phase of this study. 


\section{Conclusion}

Establishing public policies aimed at preventing SCIs should focus on older and male population. The results obtained have important implications for future public health care planning, public safety, and resource allocation. This will in turn be helpful in determining the major causes, their prevention, and subsequent management including rehabilitative measures in patient with SCI.

\section{Conflict of Interest}

None.

\section{Acknowledgments}

The authors acknowledge all the patients and their caregivers for getting data on this serious health issue.

\section{References}

1 Spinal Cord Injury. http://www.neurogenbsi.com/spinal-cordinjury-treatment-in-india/

2 Dincer F, Oflazer A, Beyazova M, Celiker R, Basgöze O, Altioklar K. Traumatic spinal cord injuries in Turkey. Paraplegia 1992;30(9):641-646
3 Kristinsdottir EA, Knutsdottir S, Sigvaldason K, Jonsson H Jr, Ingvarsson PE. [Epidemiology of spinal cord injury in Iceland from 1975 to 2014]. Laeknabladid 2016;102(11):491-496

4 Wang H, Xiang Q Li C, Zhou Y. Epidemiology of traumatic cervical spinal fractures and risk factors for traumatic cervical spinal cord injury in China.J Spinal Disord Tech 2013;26(8):E306-E313

5 Oliver M, Inaba K, Tang A, et al. The changing epidemiology of spinal trauma: a 13-year review from a Level I trauma centre. Injury 2012;43(8):1296-1300

6 Liu P, Yao Y, Liu MY, et al. Spinal trauma in mainland China from 2001 to 2007: an epidemiological study based on a nationwide database. Spine 2012;37(15):1310-1315

7 Ning GZ, Yu TQ, Feng SQ et al. Epidemiology of traumatic spinal cord injury in Tianjin, China. Spinal Cord 2011;49(3):386-390

8 Lenehan B, Street J, Kwon BK, et al. The epidemiology of traumatic spinal cord injury in British Columbia, Canada. Spine 2012;37(4):321-329

9 Knútsdóttir S, Thórisdóttir H, Sigvaldason K, Jónsson $H$ Jr, Björnsson A, Ingvarsson P. Epidemiology of traumatic spinal cord injuries in Iceland from 1975 to 2009. Spinal Cord 2012;50(2):123-126

10 Leucht P, Fischer K, Muhr G, Mueller EJ. Epidemiology of traumatic spine fractures. Injury 2009;40(2):166-172

11 Botterell EH, Jousse AT, Kraus AS, Thompson MG, WynneJones M, Geisler WO. A model for the future care of acute spinal cord injuries. Can J Neurol Sci 1975;2(4):361-380 